\title{
Honesty of Online Workers: A Field Experiment shows no Evidence of Self-Selection of Cheaters to a Cheating-enabling Work Environment
}

Marek Vranka*, Marek Hudík, Nikola Frollová, Štěpán Bahník, Markéta Sýkorová, and Petr Houdek

Prague University of Economics and Business, náměstí Winstona Churchilla 4, Prague, 13067 , Czechia.

*Corresponding author: vranka.marek@gmail.com

Declarations of interest: none

\begin{abstract}
Does the choice of an environment where cheating is possible lead to its escalation? We analyzed behavior of employees $(N=284)$ hired to perform a task online. In the manual reporting $(\mathrm{MR})$, employees could overreport the number of hours worked. In the automatic reporting (AR), the hours were counted automatically, making cheating impossible. Two-thirds of the participants were given a chance to choose the reporting scheme, the rest were assigned to the MR directly. As the actual time spent on the task was tracked in all conditions, we were able to assess the degree of overreporting by employees in MR. Although we found that people in MR slightly overreported the hours worked, employees who chose MR did not overreport their hours more than those assigned to MR at random. Moreover, participants lower in honesty-humility were not more likely to choose MR; only those higher in emotionality were. The results show that even when enabled to cheat, online workers reported their hours worked honestly and the possibility for cheaters to select cheating enabling environments may not always lead to an increase of dishonesty in organizations.
\end{abstract}

Keywords: dishonesty; self-selection; field experiment

JEL: C93; D12; D91 


\section{Introduction}

As online work becomes an increasingly common form of employment (Agrawal et al., 2015), questions about the prevalence of employees' counterproductive work behavior or cheating arise (Corrigan-Gibbs et al., 2015). The specifics of online work environments like perceived anonymity, lack of oversight, missing or weak social ties, and the abstract nature of tasks make it sometimes easier for employees to cheat; for example, by overreporting their hours worked (Burbano \& Chiles, 2020). The prevalence of cheating may further increase because of the ease with which dishonest people may self-select into environments enabling dishonest behavior. Although laboratory experiments (Akin, 2019; Brassiolo et al., 2020; Gino et al., 2013; Houdek et al., 2021) show that people more willing to cheat are in fact attracted to cheating-enabling environments, similar evidence from the field is lacking. Therefore, our field experiment in a real-world online work environment aims to explore whether employees perform their online tasks honestly and test whether the possibility of choice of cheating-enabling conditions affects the prevalence of cheating.

Most of the current evidence regarding dishonest behavior is obtained from laboratory studies (e.g., Gerlach et al., 2019; Jacobsen et al., 2017; Köbis et al., 2019; Mazar et al., 2008; Mazar \& Ariely, 2006; Rosenbaum et al., 2014; Shalvi et al., 2015) in which participants usually perform artificial, context-free tasks, unsure of their interpretation (Frollová et al., 2021) and with unclear impact of participants' behavior on their reputation (Choshen-Hillel et al., 2020).

In addition, most of the existing research ignores selection effects (Bless \& Burger, 2016; Houdek, 2019). Unlike in laboratory studies, in the real world people usually do not get randomly assigned to situations in which they can cheat. On the contrary, dishonest people are likely to look for environments where they can cheat, while honest individuals might be actively avoiding them (Brassiolo et al., 2020; Gino et al., 2013). Selection effects on moral aspects of behavior have been identified in several contexts (Houdek, 2017). For example, in countries where corruption abounds in the public sector, dishonest people are interested in working as civil servants (Banerjee et al., 2015; Hanna \& Wang, 2017). By contrast, in countries with high integrity of public service, the public positions are preferred by honest people (Barfort et al., 2019). Similarly, Cialdini et al. (2019) found that exposure to unethical leader behavior increased honest team members' likelihood of choosing to leave the team. On the other hand, individuals who engage in deception prefer to join occupations in advertising, banking, or sales (Gunia \& Levine, 2019). Individuals in real-life cheating-enabling environments are therefore likely to be different from other people due to self-selection. Consequently, the rate of cheating and factors influencing it may differ between experiments and real-world environments (Houdek et al., 2021).

Our study aims to extend the correlational and laboratory studies by conducting a field experiment that explores whether employees behave honestly when working online and tests the effect of selfselection on the rate of cheating in a real-life cheating-enabling environment. Based on the findings of previous laboratory studies, we expect that those willing to cheat will select themselves to the cheating-enabling environment and then take advantage of it:

H1: Participants will overreport more hours worked in a cheating-enabling environment if they chose it themselves than if they were assigned to it randomly. 
We also explore personality correlates of self-selection. Despite the voluminous literature regarding personal and personality characteristics associated with dishonest behavior (e.g., Gerlach et al., 2019; Heck et al., 2018), little is known about predictors of selection of cheating-enabling environments. Previous research showed a strong negative relation between the trait honesty-humility (one of HEXACO dimensions) and cheating behavior (e.g., Hilbig \& Zettler, 2015; Kleinlogel et al., 2018; Pfattheicher et al., 2019). It can be expected that honesty-humility will be related to the selection of the environment where it is possible to cheat. The preference of dishonest people to be in an environment conducive to cheating could thus be responsible for a significantly higher rate of cheating in these particular environments (Houdek et al., 2021). Therefore, we expect that:

H2: The participants who choose the cheating-enabling environment will be lower in honesty-humility than the participants who do not choose the cheating-enabling environment.

In addition, we also examine possible relations between overreporting and selection to the cheatingenabling environment and HEXACO personality traits of emotionality and conscientiousness.

\section{Methods}

\subsection{Participants}

The study was conducted in cooperation with a Prague-based IT company that creates software using machine-learning technology. From May to June 2020, 284 (56 \% female, Med $_{\text {age }}=25, I Q R_{\text {age }}=12$ ) temporary employees of the company were hired through job advertising websites, in the same way as the company usually hires such employees. The employees were hired for image annotation, that is, marking specific objects in photographs, which are then used for training of neural networks. None of the hired employees had prior experience with the company and therefore, had no reason to feel much commitment or loyalty to the company (Felfe et al., 2008; Gallagher \& Parks, 2001). Moreover, since the employees were not hired through an agency, they would not suffer reputational effects from cheating, as their behavior did not affect their future employment prospects as is usual in other forms of online temporary jobs (Kokkodis \& Ipeirotis, 2016). The after-tax pay rate was 2.2 CZK ( 0.09 USD) per minute, which corresponds to the average wage for this kind of work. For the payment, the total of reported minutes was rounded up to tens.

Employees were hired until a predetermined budget was fully spent. The final sample size has a sufficient statistical power (.80) to detect an effect of size $r=0.17$; however, some of the analyses were conducted with smaller subsets of participants, and therefore, their power was somewhat lower. The two main hypotheses were tested with a subsample of 153 participants, which has a sufficient statistical power (.80) to detect an effect of size $r=0.22$.

\subsection{Procedure}

Hired applicants received a link to an online training containing a description of the work and a way of reporting the hours worked. Two-thirds of employees were assigned to the experimental group in which they chose between manual (MR) and automatic reporting (AR). Namely, employees were told that two clients are currently requiring the image annotation they were hired to perform and the only difference is that one client asks them to record their hours worked manually and the other records the 
time actively spent on the task automatically. ${ }^{1}$ In the MR, they were asked to write a statement of the hours worked and sent it to a manager after their work was completed. As they were paid solely based on their report, the MR condition enabled them to cheat by overreporting the hours worked. In the $\mathrm{AR}$, the time spent on the task was counted automatically and the employees only confirmed the recorded hours to a manager after completing the work, therefore making cheating impossible.

Those in the control group were directly assigned to the MR, although they were informed in the online training that sometimes the reporting is done automatically, however in this case they will report their hours worked manually. Therefore, only the option to choose differed between the conditions. The time actively spent on the task (with inactivity defined as 10 or more seconds of no mouse movement) was automatically measured for all employees. ${ }^{2}$ In the MR, participants used the online timesheet to track the date, the work session, and the number of minutes they had spent on the task. The sheet enabled them to rewrite their report at any later time; these changes were recorded as well.

After receiving the introductory information, employees watched instructional videos, which explained how to perform the task and report the hours worked in detail. The videos differed for employees in the MR and AR. A series of control questions checked employees' comprehension of the instructions. After the introductory training had been completed, participants received login information necessary to access the web interface through which they performed the task. Participants in the MR also received a link to their timesheet for reporting the hours worked. After logging into the platform, participants were presented with photographs of people from real-world settings (e.g., public spaces such as a street or a shopping center) and they had to draw rectangles around persons in the photographs.

After finishing their work, participants received a questionnaire containing measures of honestyhumility, conscientiousness, and emotionality from the HEXACO scale (Ashton \& Lee, 2009) and questions about satisfaction with the job and the remuneration for work performed, frequency of similar temporary jobs and perceived difficulty of the task (see Figure 1 for the overview of the whole procedure). Voluntary completion of this questionnaire was compensated with an additional financial reward of 50 CZK ( 2.4 USD); 227 participants (i.e., $80 \%$ ) completed the questionnaire.

\footnotetext{
${ }^{1}$ See the supplementary materials at https://osf.io/57jue/ for a full transcript of the instructions provided to employees and an example of the task they performed.

${ }^{2}$ Employees were informed that the time "actively spent" on the task is measured and that they will be paid based on the time actively spent on the task, however it was not specified how the inactivity is defined.
} 


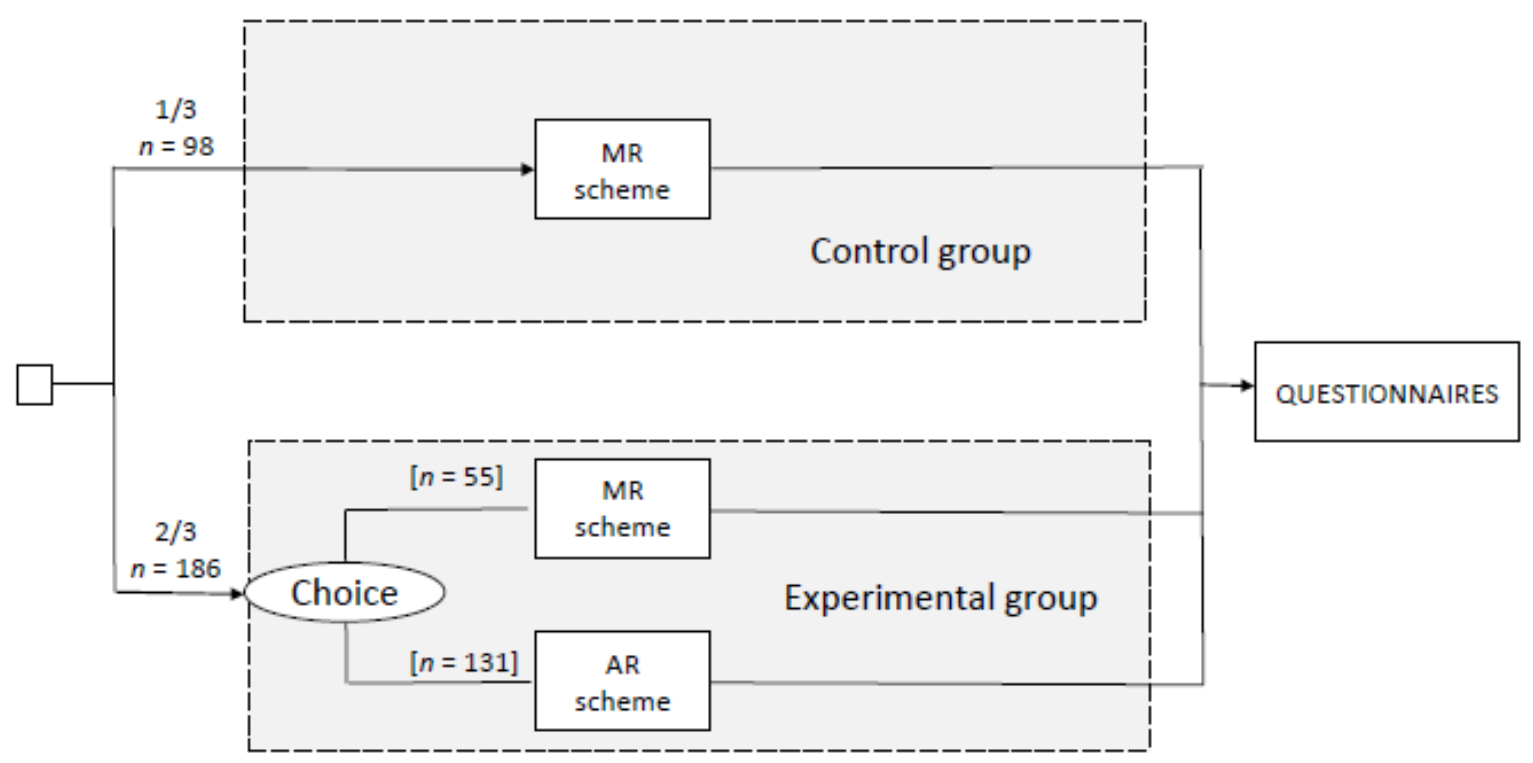

Figure 1. Schema of the study design. Participants were randomly assigned to a control and experimental group. Participants in the control group were directly assigned the MR scheme. Participants in the experimental group chose between MR and AR. At the end of the experiment, participants filled in several questionnaires.

\section{Results}

Out of 186 employees assigned to the experimental condition, 131 opted for AR, and their automatically recorded median length of work was 203 minutes $(I Q R=176)$. The remaining 55 employees opted for MR, and their automatically recorded median length of work was 247 minutes $(I Q R=183)$. For the 98 employees in the control group, the automatically recorded median length of work was 240 minutes $(I Q R=219)$. According to the Kruskal-Wallis test, there was no significant difference between any of the groups, $\chi^{2}(2)=4.528, p=0.104$.

We computed the difference between automatically recorded and reported time worked for employees who opted for or were assigned to MR $(N=153)$. Although employees significantly overreported their hours worked, $Z=2.742, p=.006$, the median overreported time was only 5 minutes (see Figure 2). Unexpectedly, underreporting was quite common; $22 \%$ of employees underreported more than 10 minutes and $16 \%$ underreported 10 minutes or less; $22 \%$ of employees overreported 10 or less minutes and the remaining $40 \%$ overreported more than 10 minutes. Only 15 participants overreported more than an hour and only 7 overreported more than two hours. 


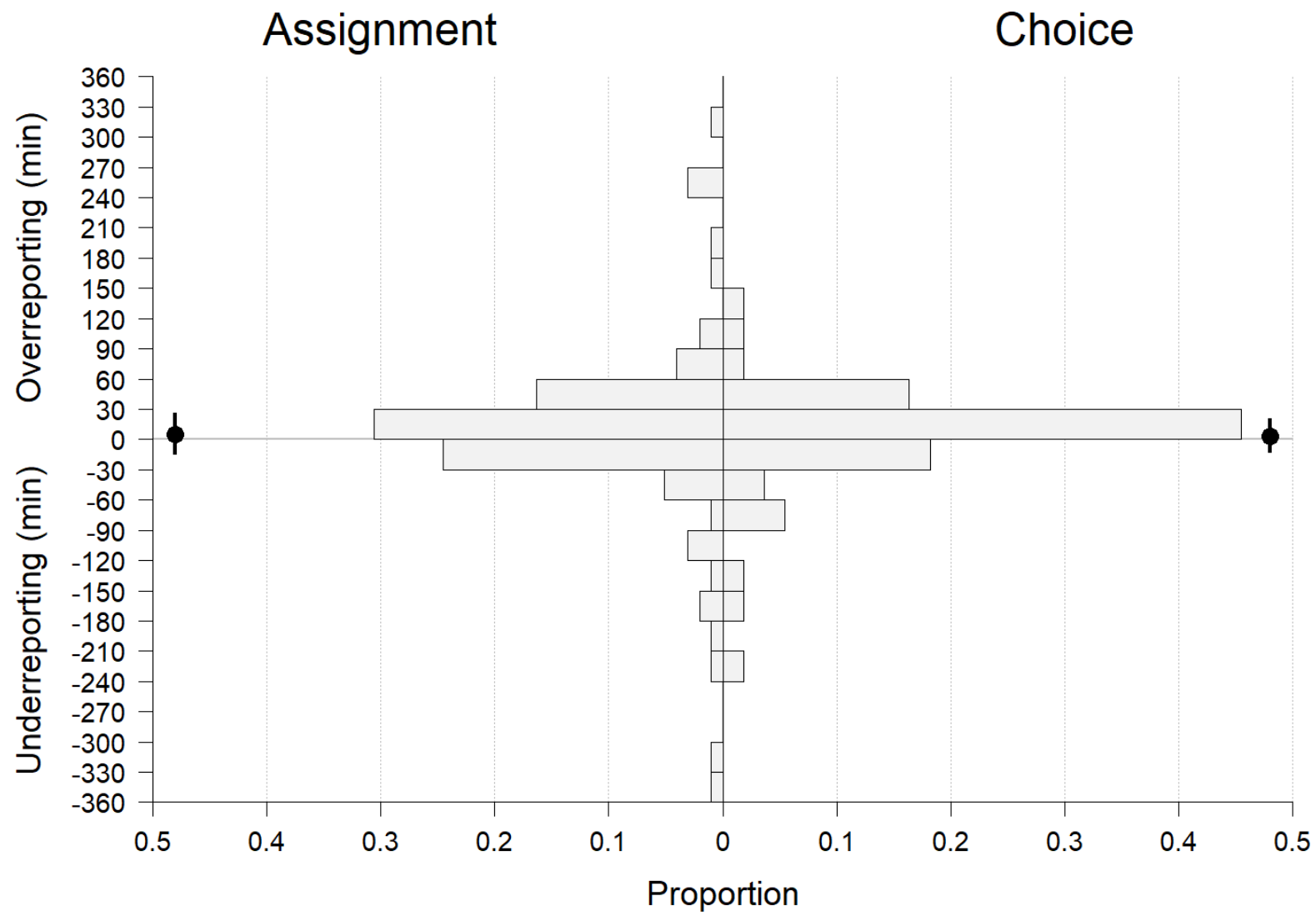

Figure 2. Overreporting of hours worked by participants with manual reporting. The histogram shows the distribution of under- and over-reporting of hours worked by employees assigned to the MR and employees who chose it themselves. The points and error bars show means and $95 \%$ confidence intervals for the means of the two groups.

Using linear regression with overreported time worked as the dependent variable and centered automatically measured time, effect-coded experimental condition, and their interaction as predictors, we found no evidence of a difference in overreporting between employees who opted for MR and those who were assigned to it, as well as no significant interaction between the condition and automatically measured time (see Table 1). We, therefore, found no support for H1.

Table 1. Overreporting predicted by condition, measured time and their interaction.

\begin{tabular}{lrcrrr}
\hline Predictors & $\mathrm{b}$ & $95 \% \mathrm{CI}$ & \multicolumn{1}{c}{$\mathrm{SE}$} & \multicolumn{1}{c}{$\mathrm{t}$} & $\mathrm{p}$-value \\
\hline chose MR condition & -4.491 & {$[-30.017 ; 21.036]$} & 12.918 & -0.348 & .729 \\
centered automatically measured time & -0.222 & {$[-0.324 ;-0.120]$} & 0.052 & -4.283 & $<.001$ \\
condition x time interaction & 0.105 & {$[-0.100 ; 0.310]$} & 0.104 & 1.015 & .312 \\
constant & 7.492 & {$[-5.271 ; 20.255]$} & 6.459 & 1.16 & .248 \\
\hline
\end{tabular}

Similarly, we found no support for $\mathrm{H} 2$, as there was no significant correlation between honestyhumility and overreporting, $r_{s}=-.113,95 \% \mathrm{CI}[-.285, .661], p=.214$, or self-selection into the MR scheme, $r_{s}=.012,95 \% \mathrm{CI}[-.148, .171] p=.833$.

Afterward, we conducted several exploratory analyses, namely Spearman correlations of emotionality, conscientiousness, work and remuneration satisfaction, and perceived work difficulty with self- 
selection into the MR and overreporting. The only variable correlated with the self-selection was emotionality, $r_{s}=.260,95 \%$ CI $[.105, .403], p=.001$; employees with higher emotionality traits were more likely to opt for manual reporting. No variables were significantly correlated with the overreported time. ${ }^{3}$

Out of 153 employees who manually reported their hours worked, only 11 took advantage of the possibility to edit their reports any time before finishing the whole task and changed their previously reported number of hours. Among those, five only decreased the previously reported time, four increased it and two both increased and decreased some of their reported times.

Lastly, we explored correlations between personality characteristics and answers about whether employees enjoyed the work, were content with the reward, and found the task difficult. More conscientious employees found the work less difficult and enjoyed it more. Those who were more honest and humbler also enjoyed the work more and were more content with their reward, the latter was true for employees higher in emotionality as well (see Table 2).

Table 2. Associations between personality traits and work perception.

\begin{tabular}{lcccc}
\hline & & Honesty-humility & Conscientiousness & Emotionality \\
\hline I enjoyed the & $r_{s}$ & $.15^{*}$ & $.24^{* *}$ & .07 \\
work. & $p$ & .021 & $<.001$ & .330 \\
\hline I am content with & $r_{s}$ & $.20^{* *}$ & .028 & $.15^{*}$ \\
the remuneration. & $p$ & .002 & 0.679 & 0.027 \\
\hline I found the work & $r_{s}$ & -.04 & $-.17^{* *}$ & .04 \\
difficult. & $p$ & .596 & .009 & .544 \\
\hline
\end{tabular}

Note. $r_{\mathrm{s}}-$ Spearman correlation coefficient.

$* p<.05 . * * p<.01$.

\section{Discussion}

On a sample of temporary employees of an IT company, we analyzed the honesty of their hours worked reporting. The hiring procedure and the performed task were identical to those used by the company in its standard operations. The work was performed online, which made cheating easier due to imperfect monitoring (Burbano \& Chiles, 2020); employees had only a temporary contract and no prior experience with the company, and therefore, possibly low commitment and loyalty to the company (Felfe et al., 2008; Gallagher \& Parks, 2001). Finally, since the employees were not hired through an agency, they would not suffer reputational effects from cheating, as their behavior did not affect their future employment prospects as is usual in other forms of online temporary jobs (Kokkodis \& Ipeirotis, 2016). Despite all these factors that should increase the likelihood of cheating, we found that employees overreported hours worked only by a few minutes on average. These results are in line with existing literature on dishonesty showing that, on average, people cheat only slightly, and that field studies are associated with even less dishonesty than laboratory studies (Gerlach et al., 2019; Procházka et al., 2021).

\footnotetext{
${ }^{3}$ See https://osf.io/57jue/ for detailed results and alternative analyses of the main hypotheses, as well as the preregistration, data, and analytical scripts.
} 
However, we also observed considerable heterogeneity in over- and under-reporting: While a tenth of workers substantially overreported the hours worked by an hour or more, about a third of employees reported a shorter time than they objectively worked. The underreporting can be explained by several reasons: they could have unwittingly omitted to count some tasks or tried not to count the time when they failed to do a quality work. Similarly, they could have tried to avoid looking like cheaters who want to overreport (Choshen-Hillel et al., 2020; Gneezy et al., 2018) and they may have also feared that their work would be examined if they reported more than the average worked hours (Hertzberg et al., 2010). Finally, it is possible that in some instances the time might have been recorded incorrectly when the annotation software was receiving false input thanks to some technical error even though employees were not working. This could likely explain the more extreme cases of underreporting. When we subtracted from the recorded work duration times, in which employees were seemingly working, but did not actually submit anything in more than 10 minutes, only $7 \%$ of employees underreported more than 10 minutes and $12 \%$ underreported 10 minutes or less. However, when this alternative measure of automatically recorded time is used, the median overreporting increases only slightly (to 8.3 minutes from 5 minutes) and results of all analyses remain qualitatively the same (see Tables S2 \& S3 in Supplementary materials).

Employees who chose the cheating-enabling environment did not cheat more than employees randomly assigned to the same environment. This result suggests that the effect of selection on cheating may not be important in the studied environment. The results of laboratory experiments (e.g., Gino et al., 2013) showing a substantial effect of selection on dishonest behavior may not be generalizable to all situations.

Our results indicate that honesty-humility is substantially related neither to the overreporting, nor the selection of a cheating-enabling environment, despite previous studies that identified honesty-humility is one of the strongest predictors of workplace deviance (Pletzer et al., 2019). A possible reason for the null findings may be that most of the overreporting was caused by honest mistakes and employees had other reasons for choosing MR than wanting to cheat. For example, they might feel uncertainty regarding whether and how much of an undeserved reward can be obtained in the cheating-enabling environment and how reporting and oversight work. Even dishonest people may have felt insecure because both reporting schemes may have seemed challenging and complicated. This explanation is indirectly supported by our finding that people with higher emotionality, who generally worry more, chose manual reporting more frequently, presumably because they did not trust the accuracy of the automatic recording.

Other associations of personality and work characteristics identified in this study are in line with previous findings (e.g., Barrick \& Mount, 1991; Johnson et al., 2011; Lee et al., 2019). Specifically, people high in honesty-humility and conscientiousness enjoyed the work more. Furthermore, conscientious people found the work less demanding. Honest and humble participants, as well as participants high in emotionality, were more satisfied with the obtained reward.

Our findings may depend on the job used, i.e., one-off work, its specific nature, and the remuneration scheme, of which the hired employees learned from instructional videos only after they applied for the job. In particular, the temporary character of work may have affected what type of individuals applied for the job. The payment for the work was low relative to the average monthly wage, and therefore, this work was possibly unattractive to the most dishonest individuals. Potentially weak selection of fraudsters into the job may explain why we did not observe selection effects in the experiment. Finally, the work took place online in the company's proprietary software. Employees could, rightly, 
fear that their behavior might be recorded even in MR. Even though cheating should still be easier in $\mathrm{MR}$, some of the less honest employees may prefer to opt for AR and then intentionally work more slowly or idle more often, thus receiving higher remuneration without the need to directly lie in their statements of hours worked. However, as the automatically recorded time did not differ between the conditions, there is no evidence to support this possibility. Future studies should verify that the same results may be achieved with long-term or recurring job tasks or experienced staff who have a detailed awareness of the nature of the work and its reporting.

\section{Conclusion}

Our field study applied an experimental design, in which workers could decide first whether to have an opportunity to cheat and then whether to actually exploit it. We found that employees overreported their hours worked, but only a little bit. Workers who chose a work-reporting system that made it easy to misreport the worked hours did not cheat more than workers randomly assigned to the same reporting system. These results suggest that the selection effect may not lead to a higher level of counterproductive work behavior because the cheaters, as measured by the honesty-humility scale, were not attracted to the cheating-enabling environment.

\section{Acknowledgements}

The research was supported by The Czech Science Foundation (GACR) Project No. 18-13766S. The funder had no role in study design, data collection and analysis, or preparation of the manuscript.

We would like to thank our outstanding research assistant Adam Kouba for providing the hiring process of employee and administrative support, and Luboš Smrčka for his assistance with the design and organization of the project. Finally, we thank the company Iterait a.s. for the opportunity to carry out our experiment on their platform. 


\section{References}

Agrawal, A., Horton, J., Lacetera, N., \& Lyons, E. (2015). Digitization and the contract labor market: A research agenda. In Economic analysis of the digital economy (pp. 219-250). University of Chicago Press.

Akin, Z. (2019). Dishonesty, social information, and sorting. Journal of Behavioral and Experimental Economics, 80, 199-210.

Ashton, M. C., \& Lee, K. (2009). The HEXACO-60: A short measure of the major dimensions of personality. Journal of personality assessment, 91(4), 340-345.

Banerjee, R., Baul, T., \& Rosenblat, T. (2015). On self selection of the corrupt into the public sector. Economics Letters, 127, 43-46.

Barfort, S., Harmon, N. A., Hjorth, F., \& Olsen, A. L. (2019). Sustaining honesty in public service: The role of selection. American Economic Journal: Economic Policy, 11(4), 96-123.

Barrick, M. R., \& Mount, M. K. (1991). The big five personality dimensions and job performance: a meta-analysis. Personnel Psychology, 44(1), 1-26. doi: 10.1111/j.1744-6570.1991.tb00688.x

Bless, H., \& Burger, A. M. (2016). A Closer Look at Social Psychologists' Silver Bullet. Perspectives on Psychological Science, 11(2), 296-308. doi: 10.1177/1745691615621278

Brassiolo, P., Estrada, R., Fajardo, G., \& Vargas, J. (2020). Self-Selection into Corruption: Evidence from the Lab. Caracas: CAF. Retrieved from http://scioteca.caf.com/handle/123456789/1567

Burbano, V., \& Chiles, B. (2020). Mitigating gig worker misconduct: Evidence from a real effort experiment. Columbia Business School Working Paper.

Choshen-Hillel, S., Shaw, A., \& Caruso, E. M. (2020). Lying to appear honest. Journal of Experimental Psychology: General, 149(9), 1719-1735. doi: 10.1037/xge0000737

Cialdini, R., Li, Y. J., Samper, A., \& Wellman, N. (2019). How bad apples promote bad barrels: Unethical leader behavior and the selective attrition effect. Journal of Business Ethics, 1-20.

Corrigan-Gibbs, H., Gupta, N., Northcutt, C., Cutrell, E., \& Thies, W. (2015). Deterring cheating in online environments. ACM Transactions on Computer-Human Interaction (TOCHI), 22(6), 123.

Felfe, J., Schmook, R., Schyns, B., \& Six, B. (2008). Does the form of employment make a difference? - Commitment of traditional, temporary, and self-employed workers. Journal of Vocational Behavior, 72(1), 81-94.

Frollová, N., Vranka, M., \& Houdek, P. (2021). A qualitative study of perception of a dishonesty experiment. Journal of Economic Methodology, 1-17. doi: 10.1080/1350178X.2021.1936598

Gallagher, D. G., \& Parks, J. M. (2001). I pledge thee my troth... contingently: Commitment and the contingent work relationship. Human Resource Management Review, 11(3), 181-208. 
Gerlach, P., Teodorescu, K., \& Hertwig, R. (2019). The truth about lies: A meta-analysis on dishonest behavior. Psychological Bulletin, 145(1), 1-44. doi: 10.1037/bu10000174

Gino, F., Krupka, E. L., \& Weber, R. A. (2013). License to cheat: Voluntary regulation and ethical behavior. Management science, 59(10), 2187-2203.

Gneezy, U., Kajackaite, A., \& Sobel, J. (2018). Lying Aversion and the Size of the Lie. American Economic Review, 108(2), 419-53.

Gunia, B. C., \& Levine, E. E. (2019). Deception as competence: The effect of occupational stereotypes on the perception and proliferation of deception. Organizational Behavior and Human Decision Processes, 152, 122-137.

Hanna, R., \& Wang, S. Y. (2017). Dishonesty and selection into public service: Evidence from India. American Economic Journal: Economic Policy, 9(3), 262-90.

Heck, D. W., Thielmann, I., Moshagen, M., \& Hilbig, B. E. (2018). Who lies? A large-scale reanalysis linking basic personality traits to unethical decision making. Judgment and Decision making, 13(4), 356.

Hertzberg, A., Liberti, J. M., \& Paravisini, D. (2010). Information and incentives inside the firm: Evidence from loan officer rotation. The Journal of Finance, 65(3), 795-828.

Hilbig, B. E., \& Zettler, I. (2015). When the cat's away, some mice will play: A basic trait account of dishonest behavior. Journal of Research in Personality, 57, 72-88.

Houdek, P. (2017). A Perspective on Research on Dishonesty: Limited External Validity Due to the Lack of Possibility of Self-Selection in Experimental Designs. Frontiers in Psychology, 8(1566), 1-6.

Houdek, P. (2019). Is Behavioral Ethics Ready for Giving Business and Policy Advice? Journal of Management Inquiry, 28(1), 48-56. doi: 10.1177/1056492617712894

Houdek, P., Bahník, Š., Hudik, M., \& Vranka, M. A. (2021). Selection Effects on Dishonest Behavior. Judgment and Decision Making, 16(2), 238-266.

Jacobsen, C., Fosgaard, T. R., \& Pascual-Ezama, D. (2017). Why do we lie? A Practical guide to the dishonesty literature. Journal of Economic Surveys. doi: 10.1111/joes.12204

Johnson, M. K., Rowatt, W. C., \& Petrini, L. (2011). A new trait on the market: Honesty-Humility as a unique predictor of job performance ratings. Personality and Individual Differences, 50(6), 857-862. doi: 10.1016/j.paid.2011.01.011

Kleinlogel, E. P., Dietz, J., \& Antonakis, J. (2018). Lucky, competent, or just a cheat? Interactive effects of honesty-humility and moral cues on cheating behavior. Personality and Social Psychology Bulletin, 44(2), 158-172.

Köbis, N. C., Verschuere, B., Bereby-Meyer, Y., Rand, D., \& Shalvi, S. (2019). Intuitive Honesty Versus Dishonesty: Meta-Analytic Evidence. Perspectives on Psychological Science, 14(5), 778-796. doi: 10.1177/1745691619851778 
Kokkodis, M., \& Ipeirotis, P. G. (2016). Reputation transferability in online labor markets. Management Science, 62(6), 1687-1706.

Lee, Y., Berry, C. M., \& Gonzalez-Mulé, E. (2019). The importance of being humble: A metaanalysis and incremental validity analysis of the relationship between honesty-humility and job performance. Journal of Applied Psychology, 104(12), 1535-1546. doi: 10.1037/apl0000421

Mazar, N., Amir, O., \& Ariely, D. (2008). The dishonesty of honest people: A theory of self-concept maintenance. Journal of marketing research, 45(6), 633-644. doi: 10.1509/jmkr.45.6.633

Mazar, N., \& Ariely, D. (2006). Dishonesty in everyday life and its policy implications. Journal of Public Policy \& Marketing, 25(1), 117-126.

Pfattheicher, S., Schindler, S., \& Nockur, L. (2019). On the impact of Honesty-Humility and a cue of being watched on cheating behavior. Journal of Economic Psychology, 71, 159-174.

Pletzer, J. L., Bentvelzen, M., Oostrom, J. K., \& de Vries, R. E. (2019). A meta-analysis of the relations between personality and workplace deviance: Big Five versus HEXACO. Journal of Vocational Behavior, 112, 369-383. doi: 10.1016/j.jvb.2019.04.004

Procházka, J., Fedoseeva, Y., \& Houdek, P. (2021). A field experiment on dishonesty: A registered replication of Azar et al. (2013). Journal of Behavioral and Experimental Economics, 90, 101617. doi: $10.1016 /$ j.socec. 2020.101617

Rosenbaum, S. M., Billinger, S., \& Stieglitz, N. (2014). Let's be honest: A review of experimental evidence of honesty and truth-telling. Journal of Economic Psychology, 45, 181-196.

Shalvi, S., Gino, F., Barkan, R., \& Ayal, S. (2015). Self-Serving Justifications: Doing Wrong and Feeling Moral. Current Directions in Psychological Science, 24(2), 125-130. doi: $10.1177 / 0963721414553264$ 